\title{
Calculating the magnetocaloric effect in second-order-type material by micromagnetic simulations: a case study on $\mathrm{Co}_{2} \mathrm{~B}$
}

\author{
Dominik Ohmer ${ }^{\mathrm{a}, *}$, Min Yi ${ }^{\mathrm{a}, \mathrm{b}, \mathrm{c}, * *}$, Maximilian Fries ${ }^{\mathrm{a}}$, Oliver Gutfleisch ${ }^{\mathrm{a}}$, Bai-Xiang Xu ${ }^{\mathrm{a}}$ \\ ${ }^{a}$ Institute of Materials Science, Technische Universitat Darmstadt, 64287 Darmstadt, Germany \\ ${ }^{b}$ State Key Lab of Mechanics and Control of Mechanical Structures 83 Key Lab for Intelligent Nano Materials and Devices of Ministry of \\ Education 83 College of Aerospace Engineering, Nanjing University of Aeronautics and Astronautics (NUAA), 210016 Nanjing, China \\ ${ }^{c}$ State Key Lab for Strength and Vibration of Mechanical Structure, Xi'an Jiaotong University, 710049 Xi'an, China
}

\begin{abstract}
We propose a calculation scheme which integrates micromagnetic simulations and the Arrott-Noakes equation for the investigation of the influence of microstructure and magnetocrystalline anisotropy on the magnetocaloric effect (MCE) in second-order magnetic phase transition materials. Using the Arrott-Noakes equation, the micromagnetic simulation results are extrapolated to temperatures above Curie temperature and applied to $\mathrm{MCE}$ calculations. With $\mathrm{Co}_{2} \mathrm{~B}$ as model material, we found that increasing magnetocrystalline anisotropy facilitates higher isothermal entropy changes $\left(\Delta S_{\mathrm{T}}\right)$. The grain size reduction results in increased $\Delta S_{\mathrm{T}}$ for constant saturation magnetization $\left(M_{\mathrm{S}}\right)$, while $\Delta S_{\mathrm{T}}$ decreases for a reduction of $M_{\mathrm{S}}$ with reduced grain size.
\end{abstract}

Keywords: Magnetocaloric effect, Micromagnetic simulation, Arrott-Noakes equation, Microstructure, Entropy change

As the global energy consumption, especially for cooling, is expected to increase, the research for more energy 30 efficient cooling devices is of high interest. Magnetic cooling devices show Carnot efficiencies of up to $60 \%$ and 5 are therefore considered as a promising alternative to conventional gas compression cooling devices. [1-3] Besides higher efficiency, magnetic cooling devices are free of re- 35 frigerant gases. Since the discovery of the giant magnetocaloric effect (MCE), [4] several promising material sys10 tems, e.g., La-Fe-Si-, [5-8] $\mathrm{Fe}_{2}$ P-based, [9-12] and Heusler alloys, $[13,14]$ have been studied extensively for potential application in MCE refrigeration. [15] In these systems, 40 MCE properties, such as the isothermal entropy change $\Delta S_{\mathrm{T}}$ and adiabatic temperature change $\Delta T_{\mathrm{ad}}$, are tun15 able by elemental substitutions.

However, there are other properties that can affect the MCE of a material system. Liu et al. [16] reported about 45 the influence of the annealing process, i.e., the microstructure, on the transition temperature and hysteresis in La-

$20 \quad$ Fe-Si alloys. Gottschall et al. [17] showed a sharpening of the phase transition in the first-order phase transition (FOPT) materials with decreasing grain size. Fries et al. [18] recently investigated the effect of magnetocrys- ${ }^{50}$ talline anisotropy on the $\mathrm{MCE}$ in $\mathrm{Co}_{2} \mathrm{~B}$.

25 Modeling of the MCE is of high interest, as a systematic experimental study of the relation between microstructure, magnetocrystalline anisotropy, and the MCE would include complex synthesis routes and annealing processes. ${ }^{55}$

\footnotetext{
*Corresponding author. E-mail: dohmer@mfm.tu-darmstadt.de

**E-mail: yimin@nuaa.edu.cn
}

There are different approaches for modeling the MCE. For FOPT materials first-principle calculations [19-21] are used to obtain exchange coupling energies and magnetic moments, which are inserted in thermomagnetic models like the Bean-Rodbell [22] model. Thermodynamic models, which describe the system by a set of macroscopic variables, can be used for FOPT [23, 24] and for secondorder phase transition (SOPT) [25-27] materials. The Arrott-Noakes equation (ANE) is an equation of state widely used for SOPT materials. [28] However, the above mentioned models cannot account for the microstructure. Magnetic microstructures can be taken into account in micromagnetic simulations. [29-31] They are based on the Landau-Lifshitz-Gilbert (LLG) equation and take the magnetocrystalline anisotropy $K$, the exchange constant $A$, and the saturation magnetization $M_{\mathrm{S}}$ as input parameters. But as $M_{\mathrm{S}}$ is zero above $T_{\mathrm{C}}$, micromagnetic simulations are generally limited to temperatures below $T_{\mathrm{C}}$.

In this work, we combine now micromagnetic simulations with the ANE for investigating the influence of microstructure and magnetocrystalline anisotropy on the $\mathrm{MCE}$ in $\mathrm{Co}_{2} \mathrm{~B}$ nanograins in the size range of 5 to $100 \mathrm{~nm}$. In doing so, we can vary the grain size, boundary thickness, and anisotropy constant $K$ and calculate $\Delta S_{\mathrm{T}}$. In detail, we propose three steps for the calculation of $\Delta S_{\mathrm{T}}$. Firstly, micromagnetic simulations with varied microstructures and magnetocrystalline anisotropy are performed for temperatures up to $T_{C}$. Secondly, the magnetization curves from the micromagnetic simulations are used to determine the parameters of ANE. Finally, the parameterized ANE is used to calculate $\Delta S_{\mathrm{T}}$ according to the 
Maxwell relations. [32]

The ANE is an equation of state, which phenomenologically describes the temperature and field dependence of the magnetization of SOPT materials in the vicinity of $T_{\mathrm{C}}$. In the ANE, the magnetization is described by

$$
\left(\frac{H}{M}\right)^{\frac{1}{\gamma}}=a\left(T-T_{\mathrm{C}}\right)+b M^{\frac{1}{\beta}}
$$

with $a$ and $b$ as characteristic constants for the material under study. The critical exponents $\beta$ and $\gamma$ can be obtained through the Kouvel-Fisher method [33] which is based on the equations

$$
\begin{gathered}
M_{0}(T) \cdot\left[\frac{d M_{0}(T)}{d T}\right]^{-1}=\frac{T-T_{\mathrm{C}}}{\beta}, \\
\chi_{0}^{-1}(T) \cdot\left[\frac{d \chi_{0}^{-1}(T)}{d T}\right]^{-1}=\frac{T-T_{\mathrm{C}}}{\gamma} .
\end{gathered}
$$

The spontaneous magnetization $M_{0}$ and initial susceptibility $\chi_{0}$ can be determined by linearly fitting the Arrott plots $\left(M^{2.5}\right.$ vs. $\left.(H / M)^{0.75}\right)$. Since micromagnetic simulations only work below $T_{\mathrm{C}}$, the direct fitting of the ANE by using micromagnetic results produces unreasonable results. To overcome this problem, we make use of the experimental data. More specifically, as $a$ and $b$ are material-specific, we make the assumption that they do not strongly depend on microstructure, and determine $a$ and $b$ by using the experimental data. The microstructure-related $\beta$ and $\gamma$ can be obtained by using the micromagnetic results and the aforementioned Kouvel-Fisher method. As a proof of concept of the proposed methodology, we take a typical SOPT material $\left(\mathrm{Co}_{2} \mathrm{~B}\right)$ as the model material.

75 Applying the Kouvel-Fisher method to the results from Fries et al. [18] and creating the corresponding Arrott plots (see Fig. 1 in supplementary), we determine $\beta$ and $\gamma$ to be ${ }^{100}$ 0.307 and 1.118, respectively. According to literature, the values for $\beta$ and $\gamma$ should be in the range of 0.4 and $4 / 3$,

80 respectively. The value $4 / 3$ corresponds to the solution for the three-dimensional Heisenberg model, [34] while 5/4 corresponds to the solution for the Ising model. [35] If $\gamma^{105}$ is closer to $5 / 4$ or $4 / 3$ than to the unity, it would imply short-range correlations in the spin-orientation, which are 85 not considered in the molecular field model. [33] Using the obtained $\beta$ and $\gamma$, we fit the experimental data with the ANE and get $a=0.379$ and $b=2.07 \cdot 10^{-17}$. With all four ${ }^{110}$ parameters obtained, we plot the ANE in comparison with the experimental data (see Fig. 2 in supplementary). Our 90 results agree well with the experimental data in Ref. [18] so that we will use the obtained $a$ and $b$ values to investigate the influence of microstructure and $K$ on the $\mathrm{MCE}$ of $\mathrm{Co}_{2} \mathrm{~B}^{115}$ nanograins.

Micromagnetic simulations are performed using the Ob${ }_{95}$ ject Oriented MicroMagnetic Framework (OOMMF). [36] We create the model geometry with a microstructure composed of $4 \times 4 \times 4$ cube-shaped $\mathrm{Co}_{2} \mathrm{~B}$ grains and straight ${ }_{120}$
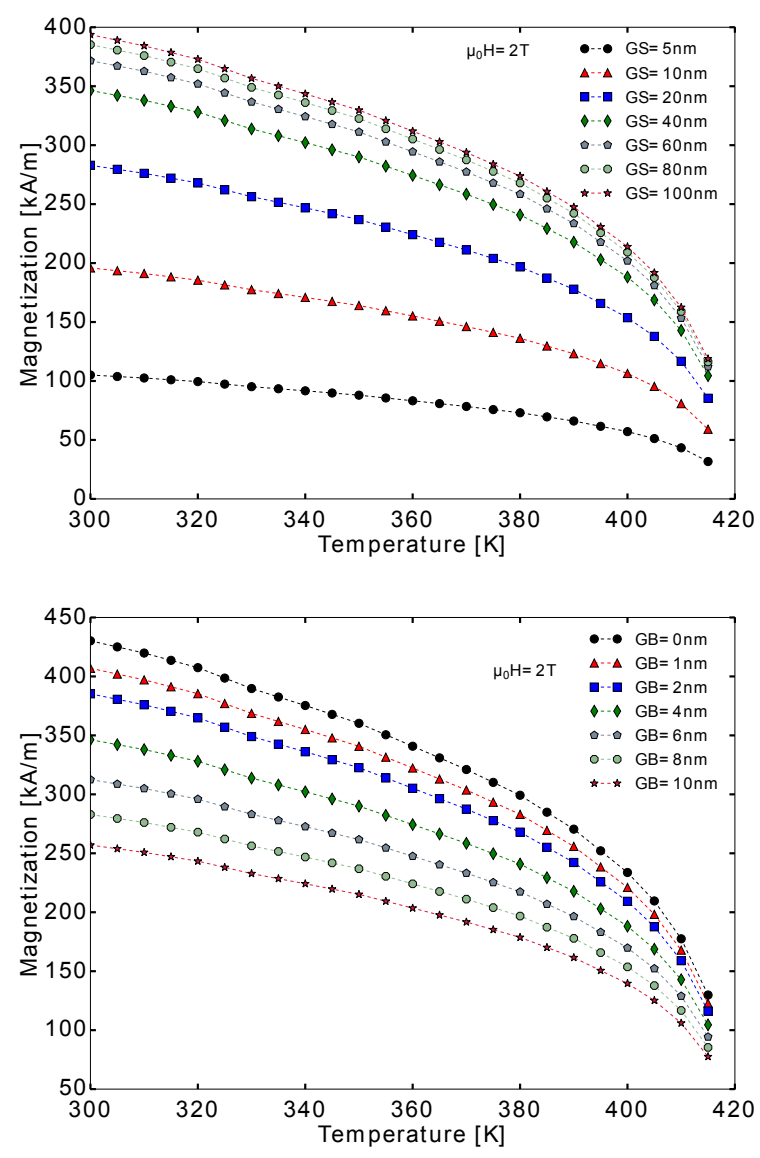

Figure 1: Simulated magnetization curves for varied grain size with a grain boundary thickness of $4 \mathrm{~nm}$ (top) and for varied grain boundary thicknesses with a grain size of $40 \mathrm{~nm}$.

grain boundaries. The grain sizes range from 5 to 100 $\mathrm{nm}$, while the grain boundary thicknesses range from 0 to $10 \mathrm{~nm}$. In all cases the mesh size is set as $2 \mathrm{~nm}$. The grain boundaries are assumed to be non-magnetic. The saturation magnetization $\left(M_{0}\right)$ and magnetocrystalline anisotropy $(K)$ of $\mathrm{Co}_{2} \mathrm{~B}$ strongly depend on temperature and are taken from the measurements by Fries et al. [18] The exchange constant $\left(A_{\mathrm{ex}}\right)$ of $\mathrm{Co}_{2} \mathrm{~B}$ is assumed to be 1 $\mathrm{pJ} / \mathrm{m}$ and in the considered range independent of temperature.

In order to calculate $\Delta S_{\mathrm{T}}$, the initial magnetization is set to be random, so that the net magnetization of the sample is zero. The magnetization process is simulated by applying an external magnetic field in the $x$-direction, which is parallel to the [100]-direction of the $\mathrm{Co}_{2} \mathrm{~B}$ grains. For temperatures higher than $100 \mathrm{~K}, \mathrm{Co}_{2} \mathrm{~B}$ exhibits the easy-plane anisotropy, spanned by the [100] and [010] direction. Thus, the magnetic field is applied parallel to an easy axis.

The magnetic field is increased from 0 up to $2 \mathrm{~T}$ by increments of $10 \mathrm{mT}$. As these simulations are isothermal, the same procedure is repeated for temperatures from 300 up to $415 \mathrm{~K}$ in increments of $5 \mathrm{~K}$. The Curie temperature 
$\left(T_{\mathrm{C}}\right)$ of $\mathrm{Co}_{2} \mathrm{~B}$ is $420 \mathrm{~K}$. The magnetization reversal process during the micromagnetic simulation can be seen in Fig. 3 in the supplementary. The resulting magnetization curves are shown in Fig. 1. As expected, the magnetization decreases for smaller grains and thicker grain boundaries. In both cases, the volume ratio of grain to grain boundary decreases, resulting in less magnetic material per unit volume.

In order to calculate $\Delta S_{\mathrm{T}}$ we need to extrapolate our micromagnetic simulation results to temperatures above $T_{\mathrm{C}}$. We apply the Kouvel-Fisher method to obtain a set of $\beta$ and $\gamma$ values for each grain size and grain boundary thickness. The trends can be seen in Fig. 2. The largest $\beta 155$ and $\gamma$ values of 0.308 and 1.35, respectively, are obtained for small grains of $5 \mathrm{~nm}$ and decrease monotonically towards 0.306 and 1.12. For the variation of grain boundary thickness, $\beta$ decreases monotonically from 0.307 for $10 \mathrm{~nm}$ to 0.305 for $0 \mathrm{~nm}$. $\gamma$ ranges between 1.12 and 1.15 with a160 minimum for $1 \mathrm{~nm}$ thick grain boundaries. However, for thicker grain boundaries $\gamma$ increases monotonically. Based on these observations, the size of the grains is expected to have a larger impact on the MCE calculations than the thickness of the grain boundary. Physically, the larger165 variation of $\gamma$ implies that the sensitivity of the system to an external field $H$ depends more on the size of the grains. Edge and corner effects are more pronounced in smaller grains, as the surface to volume ratio increases for decreasing grain size.
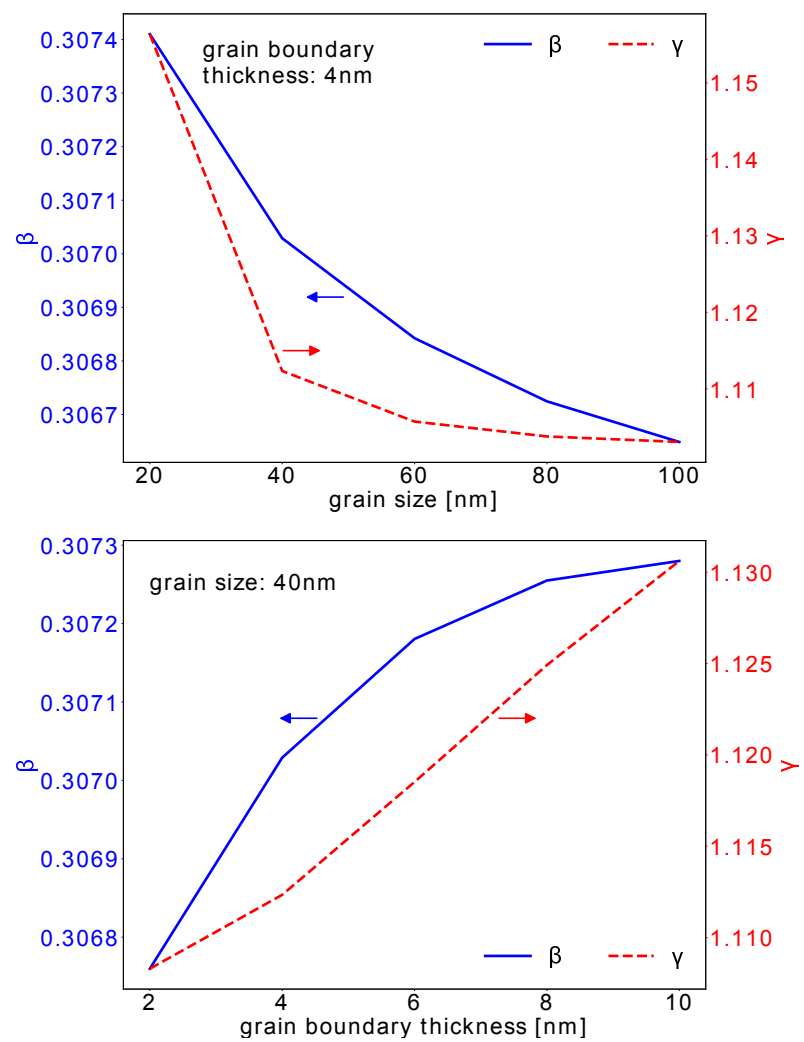

Figure 2: $\beta$ and $\gamma$ as a function of grain size (top) and grain boundary thickness (bottom).
Inserting the obtained parameters into Eq. $1, \Delta S_{\mathrm{T}}$ can be obtained by the Maxwell relation

$$
\Delta S_{T}=\int_{0}^{H_{e x t}}\left(\frac{\partial M}{\partial T}\right)_{H} d H .
$$

$\Delta S_{\mathrm{T}}$ as a function of temperature for the different microstructures is shown in Fig. 3. The inset shows the measured entropy change in poly-crystalline $\mathrm{Co}_{2} \mathrm{~B}$ and in a $\mathrm{Co}_{2} \mathrm{~B}$ single crystal in an external field of $2 \mathrm{~T}$. [18]

With the assumptions made and the simple microstructures generated, our simulation results agree well with the experimental data. We obtain $\Delta S_{\mathrm{T}}$ values between -1.7 and $-3.2 \mathrm{Jkg}^{-1} \mathrm{~K}^{-1}$ at $T_{\mathrm{C}}$, compared to $-1.4 \mathrm{Jkg}^{-1} \mathrm{~K}^{-1}$ for the single crystal measurement with the external field parallel to the easy axis and $-1.2 \mathrm{Jkg}^{-1} \mathrm{~K}^{-1}$ for the polycrystalline sample. Larger $\Delta S_{\mathrm{T}}$ values are expected as the change of magnetization with temperature in the vicinity of $T_{\mathrm{C}}$ is larger for the ANE curves (see Fig. 2). In addition, the generated microstructures are free of defects and impurities. The magnetization is free to rotate and align with the external field, resulting in larger $\left(\frac{\partial M}{\partial T}\right)_{H}$ values and thus larger theoretic entropy changes. Consequently, the calculated $\Delta S_{\mathrm{T}}$ curves show a sharper behavior around $T_{\mathrm{C}}$.

As expected from the obtained $\gamma$ values, $\Delta S_{\mathrm{T}}$ varies only slightly for different grain boundary thicknesses. The largest $\Delta S_{\mathrm{T}}$ is found for $10 \mathrm{~nm}$ grain boundaries with $1.94 \mathrm{Jkg}^{-1} \mathrm{~K}^{-1}$. For smaller boundaries $\Delta S_{\mathrm{T}}$ decreases, showing a minimum of $-1.82 \mathrm{Jkg}^{-1} \mathrm{~K}^{-1}$ for $1 \mathrm{~nm}$. This reconciles with the minimum of $\gamma$ in Fig. 2. We conclude, that the thickness of the non-magnetic grain boundary has only 175 a minor influence on the MCE and is no important parameter for its optimization.

In contrast, the variation of the grain size significantly influences the obtained $\Delta S_{\mathrm{T}}$. For larger grains $\Delta S_{\mathrm{T}}$ tends

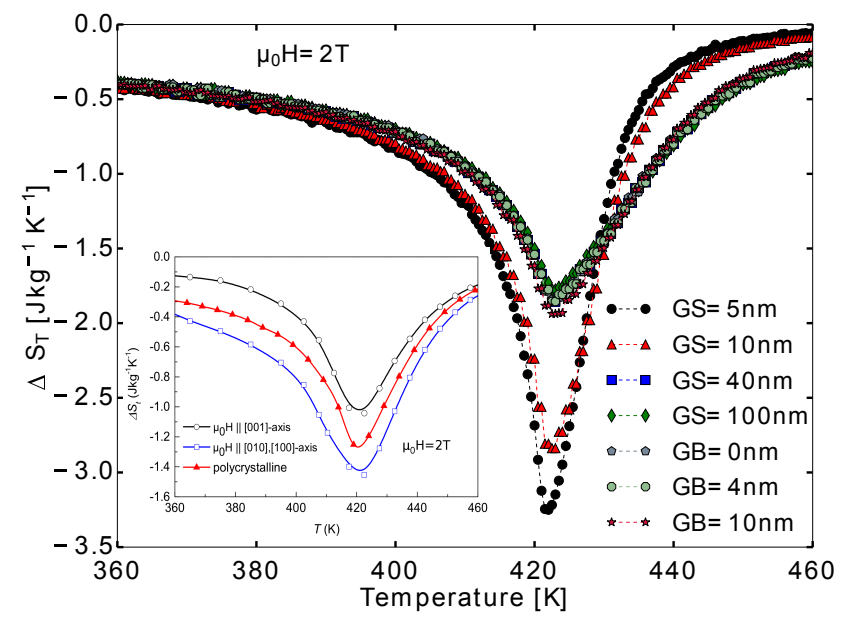

Figure 3: Specific isothermal entropy change $\Delta S_{\mathrm{T}}$ calculated via the ANE as a function of grain size and grain boundary thickness. The inset shows the experimentally measured entropy change in Ref. [18]. 
to saturate towards $-1.8 \mathrm{Jkg}^{-1} \mathrm{~K}^{-1}$, while for smaller grains ${ }_{180} \Delta S_{\mathrm{T}}$ increases, reaching $-3.25 \mathrm{Jkg}^{-1} \mathrm{~K}^{-1}$ for $5 \mathrm{~nm}$ grains. As expected from Fig. 2, the change in $\Delta S_{\mathrm{T}}$ is more significant for the variation of grain size than for the variation of boundary thickness. Due to the increased volume to surface ratio, edge and corner effects will be stronger in magnetization in smaller grains will be more sensitive to temperature, increasing $\left(\frac{\partial M}{\partial T}\right)_{H}$. Hence, we speculate that finer microstructures enhance the MCE.

Recent experiments on the relation between the partiand the $\mathrm{MCE}$ showed that $\Delta S_{\mathrm{T}}$ decreases with decreasing particle size [37-39]. However, they also reported that the magnetic properties change alongside the particle size change, e.g. smaller $M_{\mathrm{S}}$ values for smaller particles, which results in smaller $\Delta S_{\mathrm{T}}$. In our simula-

195 tion results in Fig. 3, we only consider ideal grains with the same $M_{\mathrm{S}}$. However, if we consider the significantly reduced $M_{\mathrm{S}}$ with decreased grain size, we also computationally address that smaller grains lead to smaller $\Delta S_{\mathrm{T}}$ values (see Fig. $\mathrm{xx}$ in supplementary), consistent with the experimental reports [37-39]. Therefore, with reasonable inputs, our calculation methodology is reliable.

We also calculated $\Delta S_{\mathrm{T}}$ for the external magnetic field along the hard axis (see Fig. 4 in supplementary). Consis-240 tent with the experimental results, $\Delta S_{\mathrm{T}}$ at $2 \mathrm{~T}$ is smaller compared to the case with the external magnetic field along the easy axis, as higher fields are required to fully reverse the magnetization along the hard axis and the transition is not so sharp.

Besides investigating the influence of the microstructure on the MCE, our proposed methodology can also be used to examine the influence of magnetocrystalline anisotropy $K$. That the anisotropy of the system has an influence on the MCE can be seen in the inset of Fig. 3. $\Delta S_{\mathrm{T}}$ of a $\mathrm{Co}_{2} \mathrm{~B}_{250}$ single crystal measured in an external field parallel to the easy axis is $0.4 \mathrm{Jkg}^{-1} \mathrm{~K}^{-1}$ larger than in a field parallel to the hard axis.

In the same way as for the microstructure variation, we perform micromagnetic simulations up to $415 \mathrm{~K}$ for ${ }_{255}$ scaled $K$ values that retain the relative temperature dependence. $K$ is scaled with a factor $s$ that ranges from 0 to 5 (see Fig. 4 in supplementary), where 0 corresponds to the isotropic case. We use the Kouvel-Fisher method to obtain $\beta$ and $\gamma$, and calculate $\Delta S_{\mathrm{T}}$ using Eq. 1 and Eq.260 4. $a$ and $b$ are taken as the same values as before. We are aware that varying an intrinsic property like the magnetocrystalline anisotropy may also influence the parameters $a$ and $b$. Nevertheless, we are confident to show the principle relation between the magnetocrystalline anisotropy 265 and the MCE. The results for $\Delta S_{\mathrm{T}}$ are shown in Fig. 4.

$\Delta S_{\mathrm{T}}$ is the lowest for the isotropic case $(s=0)$ and increases with larger scaling factors (i.e. larger $K$ ). This is related to the anisotropy energy which linearly depends on $K$ and therefore scales with $s$. For larger anisotropy 270 energies, the misalignment of magnetic moments with the

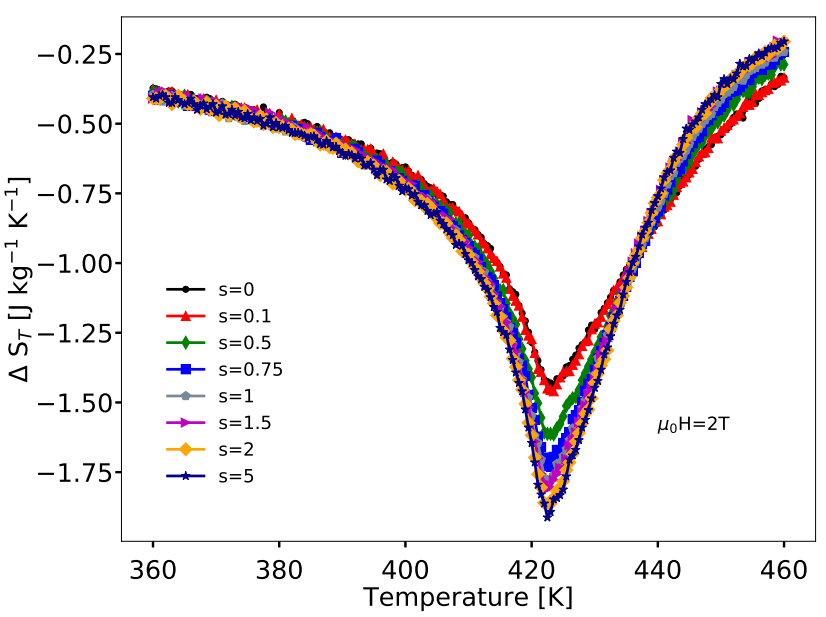

Figure 4: Comparison of $\Delta S_{\mathrm{T}}$ for scaled magnetic anisotropy constants $K$.

align faster with the external field. $\Delta S_{\mathrm{T}}$ ranges from -1.4 to $-1.95 \mathrm{Jkg}^{-1} \mathrm{~K}^{-1}$ and is found to saturate for larger $K$, showing almost identical results for $s$ equal to 2 and 5 .

In summary, we have proposed a new methodology for calculating MCE in SOPT materials from micromagnetic simulations. We combine mircomagnetic simulations with the ANE to extrapolate the simulation results to temperatures at and above $T_{\mathrm{C}}$. Experimental data is used to obtain the material-specific parameters $a$ and $b$ of ANE, while the critical exponents $\beta$ and $\gamma$ of ANE are determined by using mircomagnetic simulations and the Kouvel-Fisher method. Assuming $a$ and $b$ to be constant for the model material $\mathrm{Co}_{2} \mathrm{~B}$, we determined $\beta$ and $\gamma$ by using micromagnetic simulations results of different microstructures and anisotropy constants. $\Delta S_{\mathrm{T}}$ calculated by our methodology shows good agreement with the experimental results on $\mathrm{Co}_{2} \mathrm{~B}$. It is found that $\Delta S_{\mathrm{T}}$ increases with decreasing grain size, increasing grain boundary thickness, and increasing $K$.

Our proposed methodology is supposed to help optimizing the MCE by tuning the microstructure in SOPT materials. We are confident, that our approach can be used to predict the trend of the MCE with respect to the microstructure. This allows for a more systematic synthesis of microstructures with a predicted higher MCE. Our results imply that the MCE can be enhanced by reducing the grain size in poly-crystalline SOPT materials, when the magnetic properties of the grains are not changed by the size reduction. If $M_{\mathrm{S}}$ is significantly reduced by decreasing the grain size, smaller grains could also lead to smaller $\Delta S_{\mathrm{T}}$. In any case, results shown in this work serve as a proof of concept of our new methodology. More extensive calculations with more complex microstructures, including different grain sizes, shapes, and orientations as well as different grain boundary properties have to be performed to gain further insight on the relation between microstructure and the MCE. 


\section{Acknowledgement}

The authors acknowledge the support from the Euro275 pean Research Council (ERC) under the European Unions ${ }^{340}$ Horizon 2020 research and innovation programme (Grant agreement No 743116, project CoolInnov), the support from the German Science Foundation (DFG YI 165/11 and DFG XU 121/7-1), and the access to the Licht- ${ }^{345}$ 280 enberg High Performance Computer of Technische Universität Darmstadt. M Yi acknowledges the support of the 15th Thousand Youth Talents Program of China, the NSFC (Grant No. 11902150), the Research Fund of State ${ }^{350}$ Key Laboratory of Mechanics and Control of Mechanical 285 Structures (MCMS-I-0419G01), and A Project Funded by the Priority Academic Program Development of Jiangsu Higher Education Institutions.

\section{References}

[1] C. Zimm, A. Jastrab, A. Sternberg, V. Pecharsky, K. Gschneidner, M. Osborne, I. Anderson, in: P. Kittel (Ed.), Advances in cryogenic engineering, Springer US, Boston, MA, 1998, pp. 1759-1766. URL: https://doi.org/10.1007/ 978-1-4757-9047-4\{_\}222. doi:10.1007/978-1-4757-9047-4 -365 222.

[2] O. Gutfleisch, M. A. Willard, E. Brück, C. H. Chen, S. G. Sankar, J. P. Liu, Advanced Materials 23 (2011) 821-842. doi:10.1002/adma. 201002180.

[3] V. Franco, J. S. Blázquez, B. Ingale, A. Conde, Annual Review of Materials Research 42 (2012) 305-342. doi:10.1146/ annurev-matsci-062910-100356.

[4] V. K. Pecharsky, K. A. Gschneidner, Physical Review Letters 78 (1997) 4494-4497. URL: http://link.aps.org/doi/10.1103/ PhysRevLett.78.4494. doi:10.1103/PhysRevLett.78.4494.

[5] F. X. Hu, B. G. Shen, J. R. Sun, Z. H. Cheng, G. H. Rao, X. X. Zhang, Applied Physics Letters 78 (2001) 3675-3677. doi:10. 1063/1.1375836.

[6] A. Fujita, S. Fujieda, Y. Hasegawa, K. Fukamichi, Physical Review B - Condensed Matter and Materials Physics 67 (2003) 12. doi:10.1103/PhysRevB.67.104416.

[7] J. Lyubina, R. Schäfer, N. Martin, L. Schultz, O. Gutfleisch, Advanced Materials 22 (2010) 3735-3739. doi:10.1002/adma. 201000177.

[8] J. Liu, J. D. Moore, K. P. Skokov, M. Krautz, K. Löwe, A. Bar- ${ }_{385}$ cza, M. Katter, O. Gutfleisch, Scripta Materialia 67 (2012) 584589. doi:10.1016/j.scriptamat.2012.05.039.

[9] O. Tegus, E. Brueck, K. H. J. Buschow, F. R. de Boer, Nature 415 (202) 150. doi:10.1002/chin. 200214015.

[10] A. Yan, K. H. Müller, L. Schultz, O. Gutfleisch, Journal of ${ }_{390}$ Applied Physics 99 (2006) 0-4. doi:10.1063/1.2162807.

[11] F. Guillou, G. Porcari, H. Yibole, N. Van Dijk, E. Brück, Advanced Materials 26 (2014) 2671-2675. doi:10.1002/adma. 201304788.

[12] M. Fries, L. Pfeuffer, E. Bruder, T. Gottschall, S. Ener, L. V. B. 395 Diop, T. Gröb, K. P. Skokov, O. Gutfleisch, Acta Materialia 132 (2017) 222-229. URL: http://www.sciencedirect. com/science/article/pii/S1359645417303348. doi:https: //doi.org/10.1016/j.actamat.2017.04.040.

[13] J. Liu, T. Gottschall, K. P. Skokov, J. D. Moore, O. Gutfleisch, ${ }_{400}$ Nature Materials 11 (2012) 620-626. URL: http://dx.doi.org/ 10.1038/nmat3334. doi:10.1038/nmat3334.

[14] T. Gottschall, K. P. Skokov, D. Benke, M. E. Gruner, O. Gutfleisch, Physical Review B 93 (2016) 2-7. doi:10.1103/ PhysRevB.93.184431.

[15] J. Y. Law, V. Franco, L. M. Moreno-Ramírez, A. Conde, D. Y. Karpenkov, I. Radulov, K. P. Skokov, O. Gutfleisch, Nature Communications 9 (2018) 2680. URL: http:// www . nature.com/articles/s41467-018-05111-w. doi:10.1038/ s41467-018-05111-w.

[16] J. Liu, M. Krautz, K. Skokov, T. G. Woodcock, O. Gutfleisch, Acta Materialia 59 (2011) 3602-3611. URL: http:// dx.doi.org/10.1016/j.actamat.2011.02.033. doi:10.1016/j. actamat.2011.02.033.

[17] T. Gottschall, D. Benke, M. Fries, A. Taubel, I. A. Radulov, K. P. Skokov, O. Gutfleisch, Advanced Functional Materials 27 (2017) 1-6. doi:10.1002/adfm.201606735.

[18] M. Fries, K. P. Skokov, D. Y. Karpenkov, V. Franco, S. Ener, O. Gutfleisch, Applied Physics Letters 109 (2016) 232406. URL: http://aip.scitation.org/doi/10. 1063/1.4971839. doi:10.1063/1.4971839.

[19] B. N. Harmon, V. N. Antonov, Journal of Applied Physics 91 (2002) 9815-9820. doi:10.1063/1.1461896.

[20] D. Paudyal, V. K. Pecharsky, K. A. Gschneidner, B. N. Harmon, Physical Review B 73 (2006) 1-12. URL: https : //link.aps.org/doi/10.1103/PhysRevB.74.012403. doi:10.1103/PhysRevB.74.012403.

[21] V. D. Buchelnikov, V. V. Sokolovskiy, H. C. Herper, H. Ebert, M. E. Gruner, S. V. Taskaev, V. V. Khovaylo, A. Hucht, A. Dannenberg, M. Ogura, H. Akai, M. Acet, P. Entel, Physical Review B 81 (2010) 094411. URL: https : //link.aps .org/doi/10. 1103/PhysRevB.81.094411. doi:10.1103/PhysRevB.81.094411.

[22] C. P. Bean, D. S. Rodbell, Physical Review 126 (1962) 104-115. doi:10.1103/PhysRev.126.104.

[23] V. Basso, G. Bertotti, M. Lobue, C. P. Sasso, Journal of Magnetism and Magnetic Materials 290-291 PA (2005) 654-657. doi:10.1016/j.jmmm. 2004.11.324.

[24] Y. I. Spichkin, A. M. Tishin, Journal of Magnetism and Magnetic Materials 290-291 PA (2005) 700-702. doi:10.1016/j. jmmm. 2004.11.341.

[25] H. Oesterreicher, F. T. Parker, Journal of Applied Physics 55 (1984) 4334-4338. doi:10.1063/1.333046.

[26] A. Y. Romanov, V. P. Silin, Fizika Metallov i Metallovedenie 83 (1997) 5-11.

[27] V. Franco, A. Conde, L. F. Kiss, Journal of Applied Physics 104 (2008) 1-5. doi:10.1063/1.2961310.

[28] A. Arrott, J. E. Noakes, Physical Review Letters 19 (1967) 786789. doi:10.1103/PhysRevLett.19.786.

[29] D. C. Crew, E. Girt, D. Suess, T. Schrefl, K. M. Krishnan, G. Thomas, M. Guilot, Physical Review B - Condensed Matter and Materials Physics 66 (2002) 1-13. doi:10.1103/PhysRevB. 66.184418.

[30] A. Thiaville, J. M. García, R. Dittrich, J. Miltat, T. Schrefl, Physical Review B - Condensed Matter and Materials Physics 67 (2003) 1-12. doi:10.1103/PhysRevB.67.094410.

[31] M. Yi, O. Gutfleisch, B. X. Xu, Journal of Applied Physics 120 (2016). doi:10.1063/1.4958697.

[32] J. C. Maxwell, P. Trans, R. S. Lond, Philosophical Transactions of the Royal Society of London 155 (1865) 459512. URL: http://www.royalsocietypublishing.org/doi/10. 1098/rstl.1865.0008. doi:10.1098/rstl.1865.0008.

[33] J. S. Kouvel, M. E. Fisher, Physical Review 136 (1964). doi:10. 1103/PhysRev.136.A1626.

[34] C. Domb, M. F. Sykes, Physical Review 128 (1962) 168-173. doi:10.1103/PhysRev.128.168.

[35] C. Domb, M. F. Sykes, Journal of Mathematical Physics 2 (1961) 63-67. doi:10.1063/1.1724213.

[36] M. Donahue, D. Porter, J. Lau, R. McMichael, NIST J. Res. 114 (1999) 57-67.

[37] T. Toliński, K. Synoradzki, Acta Physica Polonica A 126 (2014) 160-161. doi:10.12693/APhysPolA.126.160.

[38] M. Kaya, I. Dincer, S. Akturk, Y. Elerman, Metallurgical and Materials Transactions A: Physical Metallurgy and Materials Science 47 (2016) 4983-4987. doi:10.1007/s11661-016-3661-x.

[39] H. Baaziz, A. Tozri, E. Dhahri, E. K. Hlil, Chemical Physics Letters 691 (2018) 355-359. URL: http://dx.doi.org/10. 1016/j.cplett.2017.10.021. doi:10.1016/j.cplett.2017.10. 021. 